\title{
The Effect of Gender and Attractiveness of Motion on Proximity in Virtual Reality
}

\author{
KATJA ZIBREK, BENJAMIN NIAY, ANNE-HÉLĖNE OLIVIER, LUDOVIC HOYET, and \\ JULIEN PETTRE, Inria Rennes \\ RACHEL MCDONNELL, Trinity College Dublin
}

In human interaction, people will keep different distances from each other depending on their gender. For example, males will stand further away from males and closer to females. Previous studies in virtual reality (VR), where people were interacting with virtual humans, showed a similar result. However, many other variables influence proximity, such as appearance characteristics of the virtual character (e.g., attractiveness). Our study focuses on proximity to virtual walkers, where gender could be recognised from motion only, since previous studies using point-light displays found walking motion is rich in gender cues. In our experiment, a walking wooden mannequin approached the participant embodied in a virtual avatar using the HTC Vive Pro HMD and controllers. The mannequin animation was motion captured from several male and female actors and each motion was displayed individually on the character. Participants used the controller to stop the approaching mannequin when they felt it was uncomfortably close to them. Based on previous work, we hypothesised that proximity will be affected by the gender of the character, but unlike previous research, the gender in our experiment could only be determined from character's motion. We also expected differences in proximity according to the gender of the participant. We additionally expected some motions to be rated more attractive than others and that attractive motions would reduce the proximity measure. Our results show support for the last two assumptions, but no difference in proximity was found according to the gender of the character's motion. Our findings have implications for the design of virtual characters in interactive virtual environments.

CCS Concepts: • Computing methodologies $\rightarrow$ Motion capture; Perception; Virtual reality;

Additional Key Words and Phrases: Perception, proximity, virtual reality, gender

ACM Reference format:

Katja Zibrek, Benjamin Niay, Anne-Hélène Olivier, Ludovic Hoyet, Julien Pettre, and Rachel McDonnell. 2020. The Effect of Gender and Attractiveness of Motion on Proximity in Virtual Reality. ACM Trans. Appl. Percept. 17, 4, Article 14 (November 2020), 15 pages.

https://doi.org/10.1145/3419985

We wish to thank all the reviewers for their comments, and the participants in our experiments. This work was funded by Science Foundation Ireland as part of the "Game Face" project (Grant 13/CDA/2135) and under the ADAPT Centre for Digital Content Technology (Grant 13/RC/2106), the French ANR JCJC Per ${ }^{2}$ project (ANR-18-CE33-0013), as well as the H2020 ICT-25 RIA PRESENT project (\#856879).

Authors' addresses: K. Zibrek, B. Niay, A.-H. Olivier, and L. Hoyet, MimeTIC, Inria, Univ Rennes, CNRS, IRISA, M2S, Campus de Beaulieu, Rennes, France, 35042; emails: \{katja.zibrek, benjamin.niay\}@inria.fr, anne-helene.olivier@irisa.fr, ludovic.hoyet@inria.fr; J. Pettre, Rainbow, Inria, Univ Rennes, CNRS, IRISA, M2S, Campus de Beaulieu, Rennes, France, 35042; email: julien.pettre@inria.fr; R. McDonnell, Trinity College Dublin, College Green 2, Dublin, Ireland; email: ramcdonn@tcd.ie.

Permission to make digital or hard copies of all or part of this work for personal or classroom use is granted without fee provided that copies are not made or distributed for profit or commercial advantage and that copies bear this notice and the full citation on the first page. Copyrights for components of this work owned by others than ACM must be honored. Abstracting with credit is permitted. To copy otherwise, or republish, to post on servers or to redistribute to lists, requires prior specific permission and/or a fee. Request permissions from permissions@acm.org.

(c) 2020 Association for Computing Machinery.

1544-3558/2020/11-ART14 \$15.00

https://doi.org/10.1145/3419985

ACM Transactions on Applied Perception, Vol. 17, No. 4, Article 14. Publication date: November 2020. 


\section{INTRODUCTION}

People maintain physical distance between each other when interacting. The size of the distance signifies our comfort with the other-friends and partners will be allowed closer in our personal space, while unfamiliar people will be kept further away. Proxemics, the research area focusing on proximity, has been the subject of many social studies (most notably, Reference [18]) in the physical world where multiple characteristics of humans (familiarity, status, age, gender, etc.) were identified as the reasons why people keep varying distances between each other.

In virtual reality (VR), proxemics has established itself as a valuable resource for creating social behaviours. For example, when designing crowds of characters, knowledge on proximity can help in creating social distances between them [14]. When interacting with a simulated virtual agent in VR, proximity was identified as an indicator of social presence [4]. With the evolving use of VR for interactive collaboration, such as workspaces and conferences, the knowledge of personal distance could help design appropriate virtual spaces. While the possible applications of proximity behaviour are to be explored, the pursuit to evaluate factors affecting proximity is also ongoing. One such factor is gender. ${ }^{1}$ Several studies observed that the gender of the approaching person as well as of the observer are both important in their interactive personal distance (see review in Reference [21]). For example, females would start feeling uncomfortable with an approaching female much sooner than males, when approached by a female. However, no consistent pattern of these interactive dyads has been found [21]. This could be because it is difficult to dissociate gender effects from other gender confounding factors of proximity, such as body size or even facial expression.

To control for these confounding factors, we can investigate the effect of gender by using only motion information. It has been shown that gender can be recognised from biological motion displayed with extremely sparse cues, such as moving dots, also known as point-light displays [33]. Furthermore, studies of biological human motion retargeted on virtual characters of different appearance $[36,58]$ showed that the underlying gender of the motion can be recognised regardless of the character's appearance. Motion is a very strong visual cue and a character's motion should match its appearance to avoid undesired effects, because people expect virtual characters to behave in a manner befitting their appearance [57]. When creating highly realistic characters, the sensitivity to artefacts in motion and/or appearance becomes even more noticeable [11].

Our study investigates the effect of gender on proximity, where gender could be inferred only by observing the motion of the virtual model. We are not aware of any previous study that would only use motion cues from gender to estimate effects on proximity. We compare our results to the ones reported in Iachini et al. [25, 26], where they found that both the gender of the participant and the virtual character affected proximity in VR. However, their study focused only on the gender based on the character's appearance. Our present study uses an androgynous model with male and female motions applied to it. Thus, we investigate if information coming from motion alone is strong enough to influence people's behaviour when interacting with virtual characters.

Other motion information was shown to influence proximity. Motion can be perceived as more or less attractive [24], and it was previously found that attractiveness plays a role in how we interpret the intent of the approaching person. The proximity distance to attractive people (regardless of gender) tends to be shorter [6, 32]. This effect has not been tested for motion alone, therefore our study makes a novel attempt at exploring the potential interaction between attractive motion and proximity in addition to gender effects.

\footnotetext{
${ }^{1}$ We chose to use the term "gender" instead of the term "sex" throughout the article for clarity purposes even though both terms are valid according to Reference [56], who interprets gender as a sociocultural construct associated with maleness and femaleness and sex as a demographic category. The most recent available guideline from the European Commission [12] makes a similar distinction between gender (social construct) and sex (biologically determined characteristic). Our research does not presuppose that the distinction of motion between males and females is of sociocultural or biological nature. We only make exceptions to the terminology when citing works of other authors (i.e., if the authors use the term "sex," we use their terminology).
} 
Our main results were:

- Participants in VR do not change their comfortable distances from characters based on the gender of the character's motion.

- Female participants require larger space to feel comfortable than males when approached by a virtual character.

- Participants keep further away from characters with unattractive walking motions than those with attractive motions.

These results allow us to discuss the implications on interactions between users and virtual characters in HMD-based VR, and in particular how proximity is affected by motion and attractiveness.

\section{BACKGROUND}

\subsection{Gender from Motion}

Gender is a fundamental characteristic that is used during everyday human social interactions. Authors considered various aspects related to gender, such as gender differences in their preferences [19], gender bias in education [2], medicine [52], or sports [13], as well as the effect of gender body swap illusion using VR [44]. Besides the question of appearance, a lot of studies investigated the recognition of gender from motion without any other cues.

People can recognise gender from walking motion even with sparse information available. Kozlowski and Cutting [33] confirmed gender could be recognised from the so-called point-light displays (PLD), a technique where patches of reflective tape are put on the joints of human walkers, and lights and camera are manipulated in such a way that only moving dots are visible. It was found that walking motion displays gender cues, where hip-sways indicate a female mover, while shoulder motion indicates a male mover [33]. Gender recognition will therefore be easier if more gender cues are present in the motion. Apart from distinct hip and shoulder movement, other factors influence gender recognition [7], including the speed of displayed walking motion (slowed-down video of a PLD walking motion was more difficult for gender recognition) and the time it takes to recognise the gender (average recognition time in the study was 4.4 seconds).

With regard to studies that have investigated the importance of appearance and motion, Mather and Murdoch [35] found that synthetically generated motion was a more salient source of sex information than the body structure. Johnson and Tassinary [29] studied the effects of both shape and motion on the perception of sex and attractiveness and found that the shape of the character was more informative of the sex of the individual than its motion, but that both form and motion contributed to participants' judgments of attractiveness [30]. Studies in computer graphics, which retargeted motion captured walks of actors of both genders to androgynous or gendered virtual characters, found that both shape and motion contribute to the recognition: When shape is obscured, motion becomes dominant and vice versa [36, 37]. An obvious mismatch in appearance and motion, such as an obviously female motion on a male character, will, however, intensify the information coming from motion [58].

\subsection{Proxemics}

Proxemics is a study of personal space and may be described as the area individuals maintain around themselves into which others cannot intrude without arousing discomfort [21]. In the context of evaluating proximity in standardised and controlled conditions, it can be measured as active (the participant approaches the character) or passive (character approaches the participant-permeability of personal space). The minimum distance (at which the participant feels comfortable in approaching the character) or stop distance (the point at which the participant does not feel comfortable with being approached by a character anymore) are the typical ways to measure proximity. 
Ecologically varied studies on proximity have been conducted in virtual environments as well. Proximity measure has been used in VR or interactive virtual environments $[4,5]$ as an indicator of social presence-people stood further away from virtual agents if they had a higher sense of them being actually there in the space with them. Proximity can therefore be used in VR as a behavioural measure of comfort with the virtual character, provided that a sufficient social presence is established. Another important condition for the reliability of this measure is that the participant has a visual representation of their body in VR [5].

An important factor of proximity is the appearance [22]. Social aspects of personal space conveyed through the appearance of an obstacle to avoid were investigated both in VR and physical reality by comparing locomotor behaviour when interacting with an anthropomorphic obstacle (i.e., a human) as opposed to inanimate objects $[16,48]$. Clearance distance was shown to be larger when interacting with an anthropomorphic obstacle. Moreover, authors reported an effect of the orientation of the human obstacle, with a larger distance for humans standing perpendicular to the participant's path [48], larger when a virtual character is facing away and closer when facing towards participants [31].

Previous findings conducted on the permeability of space in the physical world, as well as VR, report slight variations in minimum proximity distances. Hayduk [20] reports that an approaching person became slightly, moderately, and very uncomfortably close at around 70,50 , and $30 \mathrm{~cm}$, respectively. The reason for this variability is in the variety of factors influencing the minimum distance, such as culture [18], age [26], status indicated by the height of the character [4], familiarity [20], and even facial expression [8]. There is also evidence that an attractive appearance of the approaching person is allowed closer into the other person's personal space (see for example Reference [32]). Gender of the people has frequently been identified as a source of interpersonal distance variation.

\subsection{Gender Dyads and Proximity}

Studies in the physical world that were investigating interpersonal distance behaviour of people involved in interactive dyads proposed that differences exist in how males approach other males and females, while further differences exist when the approaching person is female. Brady and Walker [9] found male-to-male distance to be the largest, followed by male to female, and last, female-to-female the smallest. A similar pattern was found by Hewitt and Henley [23], with the exception that the distance between females and males invading their space was the largest, while males allowing females into their personal space resulted in the smallest distances. However, it seems difficult to establish a general rule on the nature of proximity depending on gender, especially because other studies found even more variation in the distances between the gender dyads [1,54].

Studies in virtual environments were also exploring proximity behaviour according to gender but further variation in the distance of different dyads was reported. Study in a non-immersive collaborative environment reported that an invading male in female personal space did not result in the largest distance as previously found [42]. In immersive VR experiments using the CAVE system, users were directly approached by a virtual character $[25,26]$. Distance was particularly large with participants approached by virtual males and smaller with virtual females. Also, female participants preferred further proximity from the characters than male participants. All the mentioned studies used virtual characters that appeared morphologically as females and males.

Why exactly such variations in proximity behaviour exist, whether in physical or VR, is unknown. Hayduk [21] suggests that viewing gender as a simple dichotomy is a misconception. For example, body size affects proximity and is usually confounded by gender, where males have larger body sizes than females [27]. Also, there are gender differences in the readiness to express certain emotions-males tend to more readily express anger [45], while females more frequently express fear and sadness [15]. Emotions have shown to affect personal distance, especially aggressively behaviour as reported by Hayduk [21] and angry facial expression [8]. Visual attributes that are associated with but not exclusive to the gender could therefore be the reason behind the variability of results. 
A lot of appearance attributes could be controlled by analysing the motion only. To our knowledge, there are no studies that investigated how proximity is affected specifically by human motion. As already mentioned, human motion can provide gender cues, and it can also be perceived as more or less attractive, depending on the gender [55]. One study found attractiveness of motion is independent from gender as well [24]. In this study, the elimination of distinctiveness of motion by merging different motion captured walks resulted in greater symmetry of the motion, which improved the attractiveness of the character with such motion applied. Since both gender and attractiveness affect proximity, it is likely that information coming from motion could affect proximity.

\section{OBJECTIVES AND HYPOTHESES}

We designed an experiment in HMD-based VR to further explore how proximity is affected by the motion of the virtual character. We refer to the study of Iachini et al. [26], where it was found that the gender of the character affects viewer's proximity in VR. This was achieved by exposing participants to approaching characters of different gender, and the participants needed to stop them as soon as the distance between them and the character made them feel uncomfortable. The gender of the characters in that study was manipulated by changing the appearance of the model. Our novel approach was to design a similar study, where the gender of the characters could only be inferred from their walking motion. We therefore used one androgynous virtual model to display the different walking motions of male and female actors. We also included the exploration of attractiveness of the walking motions to study its effect on proximity, an association that has not been studied before. The results of our study would show us how important motion is in the overall perception of virtual humans.

The use of an androgynous model also helped us to control for unwanted effects of other appearance attributes that could affect proximity in addition to gender (body size, facial expression). We argue that previous studies could have reached inconclusive conclusions on gender effects on proximity, because they were using either actual people or male and female virtual models. This introduced additional variation in their data, which we avoided by keeping the model constant across both male and female animations.

We formed a number of hypotheses:

H1: female walking motion applied to a virtual character will reduce the viewer's proximity to that character. With $\mathrm{H} 1$, we predict that the viewer's proximity will be affected by the gender of the character's motion. The results of Iachini et al. [26] showed that female characters were allowed further into the personal space than male characters. We expect that gender in our study, evident only from the character's walking motion, will have a similar effect.

H2: female participants will choose further proximity distances to virtual characters than male participants. As $\mathrm{H} 2$ describes, we expect proximity behaviour to be different according to the participant's gender in our experiment. Female participants will in general prefer further distances from the character than male participants, as reported by Iachini et al. [26].

H3: attractive motions will reduce the viewer's proximity to that character in comparison to lessattractive motions. It has been shown that walking motions vary in attractiveness [24], and previous work has shown that an attractive appearance affects the proximity measure (see for example Reference [32]). For this reason, we expect attractiveness of the individual motion will have an effect on proximity as well.

\section{STIMULI CREATION AND APPARATUS}

The walking animations used for this experiment were provided by the authors of Reference [24] on request, including walking motions from 20 female and 19 male actors, some of which were previously used for their experiments. Their motion-capture process was performed using a 19-camera Vicon optical tracking system, with 67 markers positioned on each actor's body. Actors practiced walking with a metronome at the speed of 

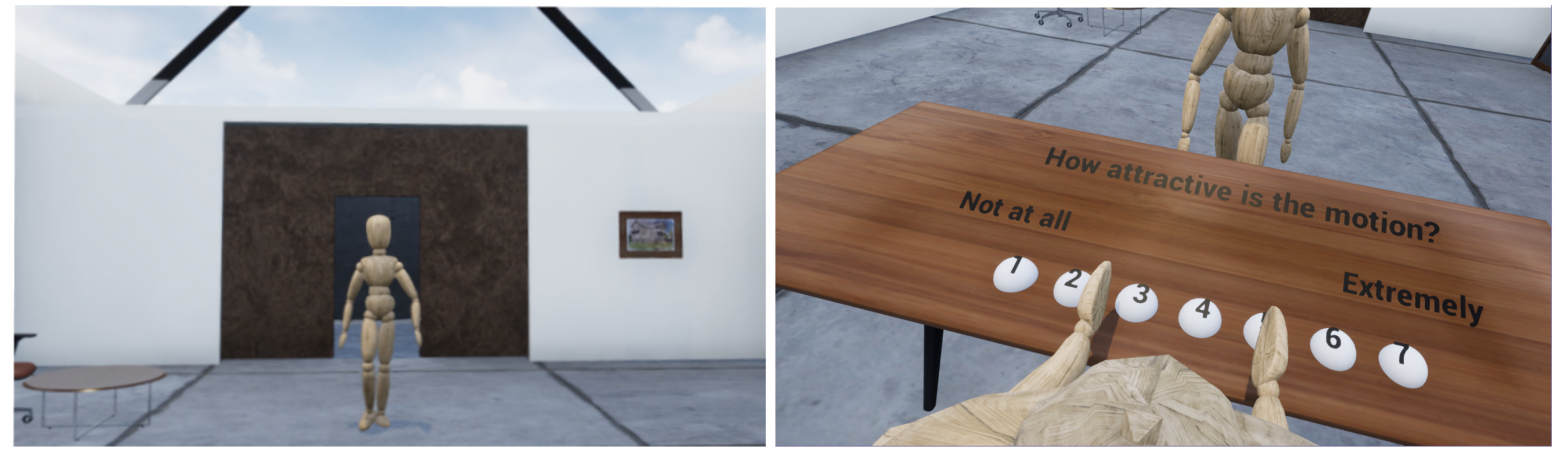

Fig. 1. Example of tasks in the experiment. Character approaching the participant in the Proximity task (left) and the rating scale in the Attractiveness task (right).

112 beats per minute (derived from the average of prerecorded comfortable walking speeds) until they could walk comfortably. To avoid any unnatural motions due to the distraction of the metronome, the actors trained with the metronome until they were at ease with the step frequency. Their walk was motion-captured without the metronome while the actors were maintaining the memorised frequency as much as possible. As the resulting captured speeds slightly varied, we further selected actors with the most similar speeds (average speed: $1.56 \mathrm{~m} / \mathrm{s}$, $S D: 0.05 \mathrm{~m} / \mathrm{s}$ ). Thus, 10 male and 10 female actors were selected for the final animations.

As our aim was to study the effect of gender and attractiveness from motion only, irrespective of visual appearance or shape, the animations were applied in Autodesk 3ds Max 2015 to a wooden mannequin that was judged as androgynous while static, but became either male or female with respective motions applied in previous work by McDonnell et al. [37, 38] (see Figure 1, left image). We chose the mannequin over a point light display, as virtual characters convey more visual information [11], which we felt was particularly important in VR. The motions were retargeted onto the mannequin's skeleton (note: actors were reasonably similar in age and body shape, to minimise retargeting errors). The individual motions and the character mesh were then exported separately to Unreal Engine 4.23. We built a simple virtual environment suitable for the VR experience to ensure a frame rate of at least $90 \mathrm{fps}$. The environment consisted of a simple room with some furniture (see Figure 1). The objects in the virtual room were added to increase the illusion of being in a place that could exist in real life (place illusion [50]).

As an important distance estimation criteria is also seeing one's own body in the environment [40, 47], we therefore embodied participants in the environment. In particular, we chose to use the same wooden mannequin as for the walking animations to avoid any influence of virtual representation differences (e.g., realistic avatar vs. androgynous wooden character) on the proximity measure. The avatar animation was driven using inverse kinematics in UE4, using the HTC Vive Pro controller positions and orientations to drive the hands of the virtual model. As we wanted to ensure that participants remained at the same position to avoid any effects on the evaluation of proximity, the legs of the character also remained planted in the same location over the course of the experiment. Their eye-level was set at the same height as the one of the approaching character, since the height of the character has an important effect on proximity [5].

For the proximity measure, we followed the design previously used in Iachini et al. [26] and Bönsch et al. [8], where the participant is approached by the character and needs to indicate the moment (by pressing a button on the controller that stops the character) when the distance between them and the character becomes uncomfortable. While other examples of proximity measure exist as well (e.g., active approach, where the participants approach the character [5]), we chose the design that made it possible to compare results with previous studies on gender and proximity interaction [26]. 
The participant's virtual body was positioned to view the virtual walkers, as they started to walk through the door opening, situated at approximately $9 m$ in front of them. This starting distance was larger than the $3 m$ distance chosen in the study of Iachini et al. [26], since it was found that 4.4 seconds is the average time to accurately assess the gender from motion [7]. We would therefore need at least $7.5 \mathrm{~m}$ for an accurate estimation of gender according to the speed of our walking characters, however, we added an extra $1.5 \mathrm{~m}$ to ensure that gender was sufficiently recognised.

\section{EXPERIMENT DESIGN}

\subsection{Participants}

Twenty participants (10 males) took part in this experiment. The participants were mostly European (17), with one participant from each of Asia, USA, and North Africa. Most participants (13) were in the 18-27 age group, while the rest were aged between 28 and 37. Nine participants were very familiar with computer-generated characters (had experience working with them), while others had seen movies and games that featured these characters. In terms of VR experience, most participants (11) had a medium level of experience (1-5 exposures) and one participant had never been exposed previously.

\subsection{Procedure}

The participants were first asked to read and sign a consent form, then to answer some questions about their demographics (age group, gender, nationality) and previous experience with VR and computer-generated characters. Then, they were told to wear a head-mounted display (HTC Vive Pro) and use the controllers to interact with a virtual character. They were shown where the necessary triggers on the controllers were that they would later use during the tasks.

The participants were presented with three separate tasks in a virtual room. The virtual room was similar across tasks, but with some slight adjustments for different user input (Figure 1). The participants were embodied in the virtual body of a wooden mannequin with the controllers moving only their virtual hands, while their virtual feet were planted on the floor. Before each task, the head-mounted display was reset to the same position and orientation so participants always had the same starting view and height throughout. They were additionally asked to stand in the position where the virtual feet were planted at all times. All tasks included the wooden mannequin walking towards them with the order of the animations being randomised for each task, with three repetitions of each walker (60 animations in total). The frontal view was chosen, since it facilitates sex classification [17]. Participants were told that when they pressed the trigger, the character would freeze for two seconds and then reappear at the original starting position (the door) and start walking towards them again.

In the first task, participants indicated by pressing the trigger on any controller as soon as the distance between them and the virtual character made them feel uncomfortable. The distance between the stopped character and the participant at the time of the trigger press was recorded as the proximity.

The second task was gender recognition of the walking motion, where participants were told that the motions were recorded using male and female actors and we were interested to know if they could identify them by using the left trigger on the controller to indicate "male" and the right controller to indicate "female." They could press the trigger as soon as they were confident in their answer.

The third task included the same character and walking motions and the participant rated the motions by how attractive they appeared to them on a scale from 1 (not attractive at all) to 7 (extremely attractive). By the term "attractive," we explained we are asking about how attractive or visually appealing the motion appeared to be in general, regardless of the gender of the walker.

After every task, the participants were given a choice to remove the headset and take a rest. After the three tasks, they were asked to answer a post-experiment questionnaire, where they answered questions about their 
Table 1. Subjective Response Questions Arranged by Questionnaire and Items

\begin{tabular}{|l|l|}
\hline Questionnaire & \multicolumn{1}{c|}{ Item } \\
\hline Place Illusion & "I had the sensation of being in an actual real place." \\
\hline Social Presence & $\begin{array}{l}\text { "It felt as if I was in the presence of another person in the room with me." } \\
\text { "The thought that the approaching character was not real crossed my mind often." } \\
\text { "The approaching character appeared to be alive." } \\
\text { "The approaching character was only a computerized image, not a living being." }\end{array}$ \\
\hline Embodiment & $\begin{array}{l}\text { "When I looked down on my body I felt like the virtual body is my own body." } \\
\text { "I felt as if the movements of the virtual body are caused by my movements." }\end{array}$ \\
\hline
\end{tabular}

Each statement was answered on a scale from 1-"Not at all" to 7-"Extremely."

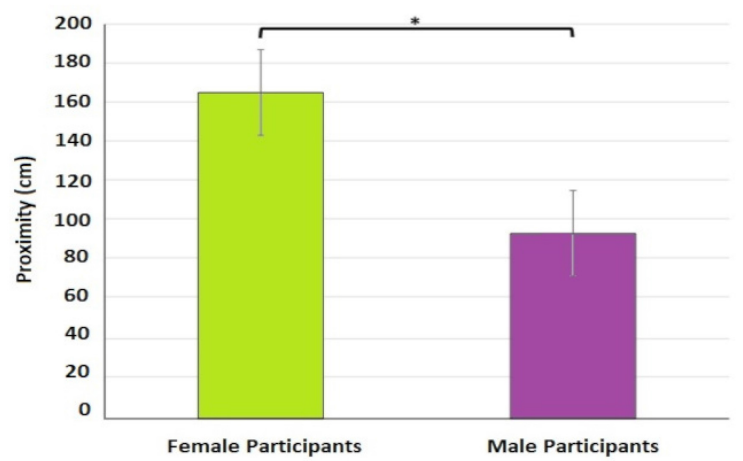

Fig. 2. Main effect of Participant Gender on proximity ratings. Error bars represent the standard error of means, and the differences marked with "*” are significant on the level of $p<0.05$.

feeling of presence in the environment and with the character, as well as the extent to which they felt the virtual body they were given was their own (see Table 1 for the questionnaire). The questions about Embodiment and Place Illusion were adapted from the studies of Slater et al. [50,51], while the Social Presence questionnaire was taken from Bailenson et al. [5].

\section{RESULTS}

\subsection{Proximity}

To analyse the results of proximity, we conducted a repeated-measure ANOVA with within-subject factors Gender (gender of the actor whose walking motion was applied to the character), Motion (10 male and 10 female actor motions), Repetitions (each motion was viewed by the participant three times), and we included the betweensubject factor Participant Gender in the analysis. All data were distributed normally (Kolmogorov-Smirnov \& Lilliefors test) and if the sphericity assumption was breached, we corrected the degrees of freedom and p-values with the Greenhouse-Geisser correction (these corrections are marked with an "*” sign next to the F-value). Post hoc tests were analysed using the Bonferroni correction. For main effects and interactions, we also include effects sizes $\left(\eta_{p}^{2}\right)$, and post hoc power estimations $(1-\beta)$.

We found no within-subject effect, but the proximity data was significantly different according to the Participant Gender $\left(F(1,18)=5.39, p=0.032, \eta_{p}^{2}=0.22,1-\beta=0.60\right)$, where female participants were stopping the character at a greater distance $\left(\bar{X}: 164 \mathrm{~cm}, \sigma_{\bar{X}}: 22.04 \mathrm{~cm}\right)$ than male participants $\left(\bar{X}: 92.48 \mathrm{~cm}, \sigma_{\bar{X}}: 22.04 \mathrm{~cm}\right)$. (See Figure 2.) 
Table 2. Gender Recognition Rates for the 10 Male and 10 Female Actor Motions

\begin{tabular}{|rrrrrrrrccc|}
\hline Gender & 1 & 2 & 3 & 4 & 5 & 6 & 7 & 8 & 9 & 10 \\
\hline Female & $88 \%$ & $68 \%$ & $55 \%$ & $98 \%$ & $88 \%$ & $97 \%$ & $53 \%$ & $85 \%$ & $88 \%$ & $25 \%$ \\
Male & $77 \%$ & $75 \%$ & $75 \%$ & $28 \%$ & $70 \%$ & $92 \%$ & $68 \%$ & $95 \%$ & $82 \%$ & $92 \%$ \\
\hline
\end{tabular}

\subsection{Gender Recognition}

We calculated the percentage of correct gender responses for each Motion (see Table 2). We see that the average recognition rate was above chance $(75 \%)$ and some motions had particularly high recognition rates (e.g., over 95\%). Two actors, one male and one female, were perceived to be of the opposite gender, signified by the low recognition rates (female motion 10 and male motion 4).

We continue to analyse the proximity data again by excluding all actors whose recognition rates were low, to determine if the lack of effect on proximity was due to the fact that within the set of 20 motions, some were poorly recognised. We chose a threshold of $70 \%$ as a reasonable estimate of the point at which gender was well recognised, and all recognition rates lower were considered not sufficient. This gave us a sample of 6 male and 6 female motions and the corresponding proximity data were analysed again using the same procedure as described in the Proximity result analysis.

The results were similar to the first proximity analysis-no effects for within-subject factors but the effect of Participant Gender was again present $\left(F(1,18)=5.23, p=0.035, \eta_{p}^{2}=0.22,1-\beta=0.60\right)$. This indicated that even when participants could perceive the gender of the motion well, there still was no effect of the gender of the walker motion on proximity.

\subsection{Attractiveness}

To evaluate if the attractiveness had an effect on people's proximity responses, we analysed the ratings for attractiveness for each of the 20 walking motions and a repeated measure ANOVA was conducted (with the betweengroups factor Participant Gender) and post hoc with the same correction was used. We found that individual motions were rated significantly different on attractiveness $\left(F(19,342)=16.084, p=0.000, \eta_{p}^{2}=0.5,1-\beta=0.99\right)$ and the post hoc showed that 8 motions ( 4 female and 4 male) were considered significantly more attractive compared to 6 other motions ( 3 male and 3 female) ( $p<0.05$, for all), while the remaining 6 motions were not significantly different from either group.

To determine if attractiveness ratings were related to proximity responses, we conducted Pearson's correlation between averaged ratings of attractiveness for each actor and the averaged proximity responses for the same actors. Both datasets showed a normal distribution. A negative correlation was found $(r=-0.61, p<0.05)$, indicating that motions that were rated higher in attractiveness had lower proximity ratings (see Figure 3 ).

\subsection{Post-questionnaire Analysis}

We also asked the participants to report their level of presence in the environment (Place Illusion) with the character (Social Presence) and the feeling of embodiment (Body Ownership). We included these results in our analysis by calculating the correlation between proximity responses to individual walking motions and the three variables, Social Presence, Place Illusion, and Embodiment. We calculated Cronbach's alpha to estimate if we could average the responses to Social Presence, which contains 5 items and Embodiment containing 2 items. Social Presence had a sufficient inter-correlation between items $(\alpha=0.76)$, therefore, we averaged over these multiple responses. The average score for Social Presence was mid-range (18.4 out of a maximum score 35; see Figure 4). For Embodiment, we found a significant difference between the ratings on the two scales $(t(20)=$ $-5.181, p=0.000$ ) (see Figure 4 for descriptive statistics). We separated the questions of Embodiment into Body 


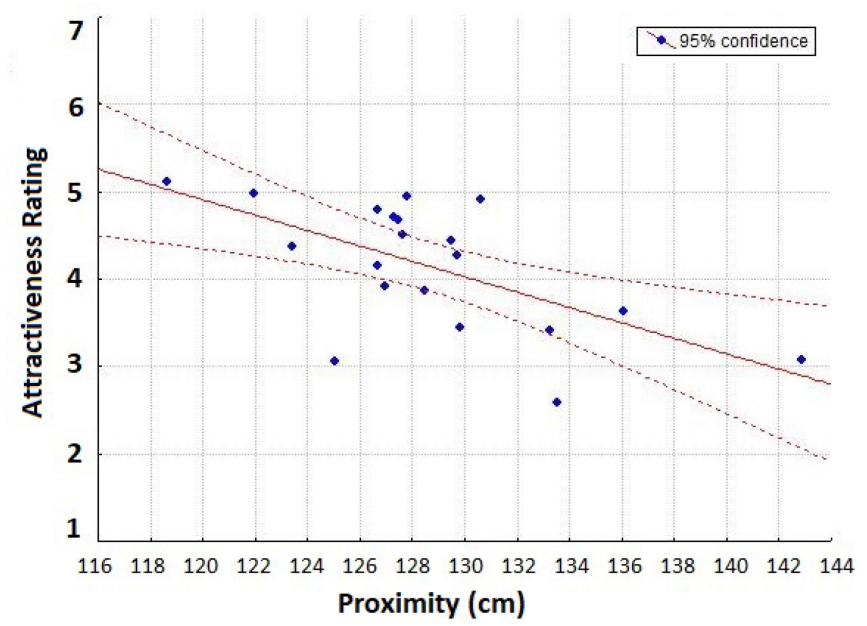

Fig. 3. Scatterplot between average attractiveness ratings and proximity responses for 20 walking motions. Graph shows a regression line describing a negative relationship between the two variables. Confidence bands of the fitted line are on the $95 \%$ confidence level.
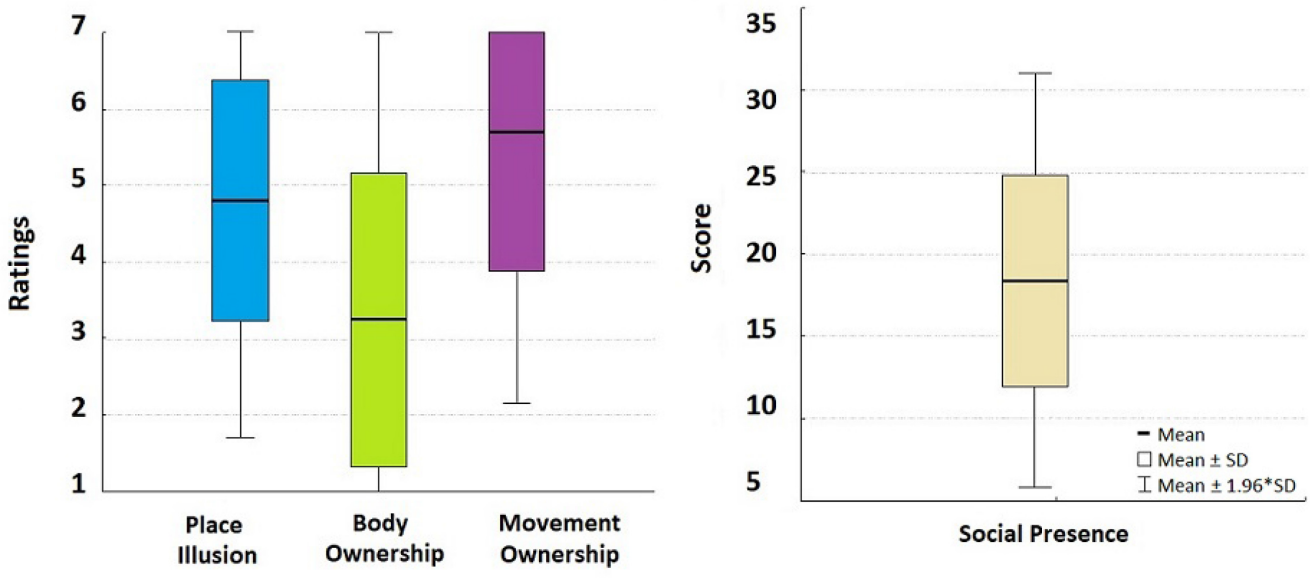

Fig. 4. Post-experiment questionnaire descriptive statistics.

Ownership and Movement Ownership, where Movement Ownership was perceived significantly higher than Body Ownership in this experiment.

We found a significant positive correlation between average proximity responses by the participants and their score on the Social Presence $(r=0.56$ and $p<0.05$,). This result shows that if a participant experienced a higher sense of presence with the character, he or she reported further proximity responses to it. Since we found an effect of the participant gender on proximity, we tested if there were differences in social presence according to the gender of participants. This was indeed the case $(t(18)=2.99, p=0.008)$, where females reported higher experienced social presence (average score $=22$ ) than males (average score $=14.8$ ). Taken together, the higher experienced social presence was related to further proximity distances, and females experienced both higher 
social presence and reported further proximity distance than males. No correlations were found between proximity and Place Illusion, Body Ownership, or Movement Ownership.

\section{DISCUSSION}

We designed a novel experiment in VR to explore the relevance of motion cues on proximity behaviour. We first predicted that a male walking motion would increase proximity while a female one would reduce it (H1). Our results did not show this effect, signifying that gender from motion alone is not enough to affect proximity. This perhaps indicates that the proximity patterns observed before in VR $[25,26]$ were more related to appearance specifics of a particular gender (such as height, facial features, clothes, etc.) and not its gender per se, or simply that gender cues of appearance are stronger than those coming from the motion. Further research studying appearance factors could help determine which characteristics of appearance are the most crucial for proximity.

We also predicted that female participants would keep further distances from the walkers than male participants (H2). We did find support for this claim, which is similar to the previous evidence, showing that females in general keep larger distances to virtual characters, regardless of the gender or anthropomorphism [25]. The fact that we found the same result is a confirmation that our proximity measure was accurate and that the absence of the expected effect in $\mathrm{H} 1$ was not due to an inappropriate method. It also shows that even simple scenarios in VR can induce a realistic behavioral reaction.

We also explored a possible link between attractiveness and proximity $(\mathrm{H} 3)$. In the physical world, attractive people are allowed closer into our personal space [6,32]. Our results in VR showed that attractive characters were allowed to approach closer as well. The additional novelty of our study is that the attractiveness ratings were based only on the motion cues, signifying the importance of motion in perception of virtual humans.

Another confirmation that our proximity measure was valid is related to the results of social presence-if the participants felt greater social presence, they indicated further proximity distance on average from the characters. This relationship was previously studied extensively by Bailenson et al. [3, 5]. An interesting result was also the difference in the social presence score according to participant gender. This raises a question: Were the gender differences in proximity we observed related to higher experienced social presence or gender differences, similar to the ones observed in the physical world? Future work is needed to explore these possibilities.

There are limitations to our study. The participant sample was relatively small. The post hoc power tests showed low power for some interactions and effects, including the effect of gender from motion. While post hoc power tests are not always reliable [34], a larger sample could potentially identify the effect of gender from motion that we initially expected to find. We therefore cannot make a strong conclusion that gender from motion does not affect proximity. Future studies using a larger sample are therefore needed. It is also worth mentioning that the current COVID-19 pandemic affected the collection of our results in a negative way, as we were not able to get a sufficient sample of participants. As a further complication, participants tested during and even postpandemic could have a different proximity response due to the new norm in personal distance guidelines when meeting with other people. Therefore, joining participants before and after the pandemic into the same study might be questionable. It would be interesting to compare, however, if there would be significant differences in the proximity behaviour pre- and post-pandemic.

Our sample also included mainly participants from Europe, which have similar cultural background related to social interaction. A sample of people from different cultural backgrounds could reveal alternative patterns, as previous research suggested [21]. It is also not clear whether the proximity difference according to gender from motion would emerge if we used slower speeds. When compared to the previous work of Iachini et al. [26], the walking speeds of the characters in our experiment were faster (Iachini used a speed of $0.5 \mathrm{~m} / \mathrm{s}$ ). However, studies from biomechanics and motor control demonstrated that human gait pattern changes at walking speeds slower than $0.6 \mathrm{~m} / \mathrm{s}$ [41, 49]. Thus, we used higher walking speeds than Iachini et al. [26] to keep a gait pattern associated with free walking (which vary from $1.2 \mathrm{~m} / \mathrm{s}$ to $1.6 \mathrm{~m} / \mathrm{s}$ in healthy young adults). We also needed to achieve a reliable gender recognition rate, and slower walking would introduce gender recognition errors [7]. 
It is important to note that the reported proximity distances may not be comparable with distances observed in the physical or even VR environments that use a different display to ours (HTC Vive Pro HMD). There is also no known difference in the way gender might affect distance perception, which could account for our result, while differences in gender are known to affect multi-sensory spatial judgments [43]. In addition to the gender specifics of perception, there could have been other factors affecting proximity. For example, a recent publication [53] showed that motion conveys personality traits, particularly perceived confidence, which could have an effect on proximity but which we did not control for in our study. However, we tried to minimise these effects by including a varied corpus of walking motions, as well as by limiting the effect of other confounding factors, such as height, speed, and non-motion visual attributes on the proximity measure in our experiment.

Ratings on social presence and place illusion were mid-range, so it is possible that a higher sense of presence with the character and in the environment could increase the sensitivity of the proximity measure and perhaps have an effect on our investigated variables. We also found a difference between movement and body ownership, which potentially impaired the overall sense of embodiment. This was perhaps due to the unrealistic body the participants were embodied in. A more realistic character could increase the level of body ownership and potentially affect the interaction with the character.

One could argue that sexual orientation of the participant might affect how they rate the attractiveness of motions. We did ensure to explain to the participants that we were interested in the overall visual attractiveness of the motions, with the expectation that they would rate motions regardless of their sexual orientation, however, we cannot completely discount for that effect. It is also important to note that there have been considerable changes in the way society understands gender. A binary classification of gender to male and female invites a reductionist view [10]. In our future work, we will consider using continuous scales to assess the gender dimension. While our research builds on previous work where a binary approach was adopted, we also recognise the need to expand and update our research methods to accommodate this development.

\section{APPLICATIONS AND FUTURE WORK}

The results of this study have practical implications for the design of virtual humans. For example, in virtual crowds, avoidance behaviour of the virtual confederate could be adapted depending on the gender of the user to allow a comfortable interpersonal distance. According to our result, the gender of the user would influence proximity to virtual characters in an immersive environment. Additionally, our results show attractive motions of a virtual character reduce proximity between them and the user. Higher attractiveness, achieved by improving motion symmetry [24], could therefore be beneficial for increasing the comfort with the character in an interactive VR scenario. While understanding the perception of motion can improve comfort with the character in VR, it can also provide guidelines for creating realistic interactions between virtual humans for non-immersive applications, such as games and other animated media. The scope of our study can therefore extend beyond VR.

Future studies could investigate the effect of different character models on the proximity and gender relationship. The models could represent females and males, with a varied set of feminine or masculine traits. Alternative proximity measures could also be included, since in the current experiment the participant was not moving through the virtual space, yet walking through the environment provides important distance cues (see for example Reference [39]). Therefore, participants' body motions could be tracked as they move towards or avoid the character to retrieve additional information about the proximity behaviour when interacting with virtual characters.

Another interesting future study could try to improve real-life distance estimations (for an overview see Reference [46]). For example, we could replicate the experiment in a virtual replica of the lab environment, as this improves real-life distance estimation as proposed by Interrante el al. [28]. We are confident, however, that even by using relative distances, the current study provides reliable evidence of the effect of motion of virtual characters on people's proximity behaviour in a VR environment.

ACM Transactions on Applied Perception, Vol. 17, No. 4, Article 14. Publication date: November 2020. 


\section{CONCLUSION}

This study investigated the effect of human motion on proximity in VR. Specifically, we were interested if people's comfort with the proximity to the approaching character of androgynous appearance would change, depending on whether the animation applied to it was female or male, and how attractive the motion was considered to be. Our results showed that the gender of motion did not have an effect on proximity, but female observers prefer larger distances between them and the character than male observers. We also found that regardless of gender, higher attractiveness of motion reduced the personal distance. We have discussed the implications for the design of interactive virtual environments and have shown the importance of motion in character design.

\section{REFERENCES}

[1] S. M. S Ahmed. 1979. Invasion of personal space: A study of departure time as affected by sex of the intruder, sex of the subject, and saliency condition. Percept. Motor Skills 49, 1 (1979), 85-86.

[2] Ann Strong Anthony. 2004. Gender bias and discrimination in nursing education: Can we change it? Nurse Educator 29, 3 (2004), $121-125$.

[3] Jeremy N. Bailenson, Eyal Aharoni, Andrew C. Beall, Rosanna E. Guadagno, Aleksandar Dimov, and Jim Blascovich. 2004. Comparing behavioral and self-report measures of embodied agents' social presence in immersive virtual environments. In Proceedings of the 7th Annual International Workshop on PRESENCE. 1864-1105.

[4] Jeremy N. Bailenson, Jim Blascovich, Andrew C. Beall, and Jack M. Loomis. 2001. Equilibrium theory revisited: Mutual gaze and personal space in virtual environments. Pres.: Teleop. Virtual Environ. 10, 6 (2001), 583-598.

[5] Jeremy N. Bailenson, Jim Blascovich, Andrew C. Beall, and Jack M. Loomis. 2003. Interpersonal distance in immersive virtual environments. Person. Social Psychol. Bull. 29, 7 (2003), 819-833.

[6] George Banziger and Renée Simmons. 1984. Emotion, attractiveness, and interpersonal space. f. Social Psychol. 124, 2 (1984), 255-256.

[7] Catharine D. Barclay, James E. Cutting, and Lynn T. Kozlowski. 1978. Temporal and spatial factors in gait perception that influence gender recognition. Percept. Psychophys. 23, 2 (1978), 145-152.

[8] Andrea Bönsch, Sina Radke, Heiko Overath, Laura M. Asché, Jonathan Wendt, Tom Vierjahn, Ute Habel, and Torsten W. Kuhlen. 2018. Social VR: How personal space is affected by virtual agents' emotions. In Proceedings of the IEEE Conference on Virtual Reality and 3D User Interfaces (VR'18). IEEE, 199-206.

[9] Adele T. Brady and Michael B. Walker. 1978. Interpersonal distance as a function of situationally induced anxiety. Brit. F. Social Clin. Psychol. 17, 2 (1978), 127-133.

[10] Jessica J. Cameron and Danu Anthony Stinson. 2019. Gender (mis) measurement: Guidelines for respecting gender diversity in psychological research. Social Person. Psychol. Compass 13, 11 (2019), e12506.

[11] Thierry Chaminade, Jessica Hodgins, and Mitsuo Kawato. 2007. Anthropomorphism influences perception of computer-animated characters' actions. Social Cog. Affect. Neurosci. 2, 3 (2007), 206-216.

[12] European Commission. 2014. Gender in EU-funded Research. Retrieved from https://op.europa.eu/fr/publication-detail/-/publication/ c118ea10-58fa-4173-a2c4-65c746918c20.

[13] Susan Tyler Eastman and Andrew C. Billings. 2000. Sportscasting and sports reporting: The power of gender bias. J. Sport Social Iss. 24, 2 (2000), 192-213.

[14] Cathy Ennis and Carol O’Sullivan. 2012. Perceptually plausible formations for virtual conversers. Comput. Animat. Virt. Worlds 23, 3-4 (2012), 321-329.

[15] Agneta H. Fischer, Patricia M. Rodriguez Mosquera, Annelies E. M. Van Vianen, and Antony S. R. Manstead. 2004. Gender and culture differences in emotion. Emotion 4, 1 (2004), 87.

[16] Amy L. Hackney, Michael E. Cinelli, and James S. Frank. 2015. Does the passability of apertures change when walking through human versus pole obstacles? Acta Psychol. 162 (2015), 62-68.

[17] A. Halevina and N. F. Troje. 2007. Sex-classification of point-light walkers: Viewpoint, structure, kinematics. In Poster Presented at Vision Science Society Meeting.

[18] Edmund T. Hall. 1969. The Hidden Dimension. Anchor Books: Doubleday, Garden City, N.Y.

[19] Tilo Hartmann and Christoph Klimmt. 2006. Gender and computer games: Exploring females' dislikes. J. Comput.-mediat. Commun 11, 4 (2006), 910-931.

[20] Leslie A. Hayduk. 1981. The permeability of personal space. Canad. J. Behav. Sci./Rev. Canad. Sciences Comport. 13, 3 (1981), 274.

[21] Leslie A. Hayduk. 1983. Personal space: Where we now stand. Psychol. Bull. 94, 2 (1983), 293.

[22] Heiko Hecht, Robin Welsch, Jana Viehoff, and Matthew R. Longo. 2019. The shape of personal space. Acta Psychol. 193 (2019), 113-122.

[23] Jay Hewitt and Rebecca Henley. 1987. Sex differences in reaction to spatial invasion. Percept. Motor Skills 64, 3 (1987), 809-810.

[24] Ludovic Hoyet, Kenneth Ryall, Katja Zibrek, Hwangpil Park, Jehee Lee, Jessica Hodgins, and Carol O’Sullivan. 2013. Evaluating the distinctiveness and attractiveness of human motions on realistic virtual bodies. ACM Trans. Graph. 32, 6 (2013), 204. 
[25] Tina Iachini, Yann Coello, Francesca Frassinetti, and Gennaro Ruggiero. 2014. Body space in social interactions: A comparison of reaching and comfort distance in immersive virtual reality. PloS One 9, 11 (2014), e111511.

[26] Tina Iachini, Yann Coello, Francesca Frassinetti, Vincenzo Paolo Senese, Francesco Galante, and Gennaro Ruggiero. 2016. Peripersonal and interpersonal space in virtual and real environments: Effects of gender and age. f. Environ. Psychol. 45 (2016), 154-164.

[27] William Ickes and Richard D. Barnes. 1977. The role of sex and self-monitoring in unstructured dyadic interactions. f. Person. Social Psychol. 35, 5 (1977), 315.

[28] Victoria Interrante, Brian Ries, and Lee Anderson. 2006. Distance perception in immersive virtual environments, revisited. In Proceedings of the IEEE Virtual Reality Conference (VR'06). IEEE, 3-10.

[29] K. L. Johnson and L. G. Tassinary. 2005. Perceiving sex directly and indirectly: Meaning in motion and morphology. Psychol. Sci. 16, 11 (2005), 890-897.

[30] K. L. Johnson and L. G. Tassinary. 2007. Compatibility of basic social perceptions determines perceived attractiveness. Proc. Nat. Acad. Sci. 104, 12 (2007), 5246-5251.

[31] Edgard Jung, Kohske Takahashi, Katsumi Watanabe, Stephan de la Rosa, Martin V. Butz, Heinrich H. Bülthoff, and Tobias Meilinger. 2016. The influence of human body orientation on distance judgments. Front. Psychol. 7 (2016), 217.

[32] Cynthia Kmiecik, Paula Mausar, and George Banziger. 1979. Attractiveness and interpersonal space. J. Social Psychol. 108, 2 (1979), $277-278$

[33] Lynn T. Kozlowski and James E. Cutting. 1977. Recognizing the sex of a walker from a dynamic point-light display. Percept. Psychophys. 21, 6 (1977), 575-580.

[34] Daniel Lakens. 2014. Observed power, and what to do if your editor asks for post-hoc power analyses. Retrieved from http:// daniellakens.blogspot.com/2014/12/observed-power-and-what-to-do-if-your.html.

[35] G. Mather and L. Murdoch. 1994. Gender discrimination in biological motion displays based on dynamic cues. Proc. Roy. Soc. Lond. Series B: Biol. Sci. 258, 1358 (1994), 273-279.

[36] Rachel McDonnell, Sophie Joerg, Jessica K. Hodgins, Fiona Newell, and Carol O'Sullivan. 2009. Evaluating the effect of motion and body shape on the perceived sex of virtual characters. ACM Trans. Appl. Percept. 5, 4 (2009), 20:1-20:14.

[37] Rachel McDonnell, Sophie Jörg, Jessica K. Hodgins, Fiona Newell, and Carol O’Sullivan. 2007. Virtual shapers \& movers: Form and motion affect sex perception. In Proceedings of the 4th Symposium on Applied Perception in Graphics and Visualization (APGV'07). 7-10.

[38] Rachel McDonnell, Sophie Jörg, Jessica K. Hodgins, Fiona Newell, and Carol O'Sullivan. 2009. Evaluating the effect of motion and body shape on the perceived sex of virtual characters. ACM Trans. Appl. Percept. 5, 4 (2009), 20.

[39] Betty J. Mohler, Sarah H. Creem-Regehr, and William B. Thompson. 2006. The influence of feedback on egocentric distance judgments in real and virtual environments. In Proceedings of the 3rd Symposium on Applied Perception in Graphics and Visualization. ACM, 9-14.

[40] Betty J. Mohler, Sarah H. Creem-Regehr, William B. Thompson, and Heinrich H. Bülthoff. 2010. The effect of viewing a self-avatar on distance judgments in an HMD-based virtual environment. Pres.: Teleop. Virt. Environ. 19, 3 (2010), 230-242.

[41] Rumi Murakami and Yohei Otaka. 2017. Estimated lower speed boundary at which the walk ratio constancy is broken in healthy adults. 7. Phys. Ther. Sci. 29, 4 (2017), 722-725.

[42] Nasser Nassiri, Norman Powell, and David Moore. 2010. Human interactions and personal space in collaborative virtual environments. Virt. Real. 14, 4 (2010), 229-240.

[43] Yoko K. Naylor and Michael K. McBeath. 2008. Gender differences in spatial perception of body tilt. Percept. Psychophys. 70, 2 (2008), 199-207.

[44] Tabitha C. Peck, My Doan, Kimberly A. Bourne, and Jessica J. Good. 2018. The effect of gender body-swap illusions on working memory and stereotype threat. IEEE Trans. Visualiz. Comput. Graph. 24, 4 (2018), 1604-1612.

[45] E. Ashby Plant, Janet Shibley Hyde, Dacher Keltner, and Patricia G. Devine. 2000. The gender stereotyping of emotions. Psychol. Wom. Quart. 24, 1 (2000), 81-92.

[46] Rebekka S. Renner, Boris M. Velichkovsky, and Jens R. Helmert. 2013. The perception of egocentric distances in virtual environments-A review. ACM Comput. Surv. 46, 2 (2013), 23.

[47] Brian Ries, Victoria Interrante, Michael Kaeding, and Lee Anderson. 2008. The effect of self-embodiment on distance perception in immersive virtual environments. In Proceedings of the ACM Symposium on Virtual Reality Software and Technology. ACM, 167-170.

[48] Ferran Argelaguet Sanz, Anne-Hélène Olivier, Gerd Bruder, Julien Pettré, and Anatole Lécuyer. 2015. Virtual proxemics: Locomotion in the presence of obstacles in large immersive projection environments. In Proceedings of the IEEE Virtual Reality Conference (VR'15). IEEE, 75-80.

[49] Noruto Sekiya, Hiroshi Nagasaki, Hajime Itoh, and Taketo Furuna. 1996. The invariant relationship between step length and step rate during free walking. F. Hum. Mov. Stud. 30 (1996), 241-257.

[50] Mel Slater. 2009. Place illusion and plausibility can lead to realistic behaviour in immersive virtual environments. Philos. Trans. Roy. Soc. B: Biol. Sci. 364, 1535 (2009), 3549-3557.

[51] Mel Slater, Bernhard Spanlang, Maria V. Sanchez-Vives, and Olaf Blanke. 2010. First person experience of body transfer in virtual reality. PloS One 5, 5 (2010), e10564.

ACM Transactions on Applied Perception, Vol. 17, No. 4, Article 14. Publication date: November 2020. 
[52] Javeed Sukhera and Chris Watling. 2018. A framework for integrating implicit bias recognition into health professions education. Acad. Med. 93, 1 (2018), 35-40.

[53] Anne Thaler, Andreas Bieg, Naureen Mahmood, Michael J. Black, Betty J. Mohler, and Nikolaus F. Troje. 2020. Attractiveness and confidence in walking style of male and female virtual characters. In Proceedings of the IEEE Conference on Virtual Reality and 3D User Interfaces Abstracts and Workshops (VRW'20). IEEE, 679-680.

[54] David R. Thomas. 1973. Interaction distances in same-sex and mixed-sex groups. Percept. Motor Skills 36, 1 (1973), 15-18.

[55] Nikolaus F. Troje. 2003. Cat walk and western hero-motion is expressive. IGSN Report (2003), 40-43. Retrieved on October 2020 from http://www.academia.edu/download/30797311/IGSN_Troje2003.pdf.

[56] Rhoda K. Unger. 1979. Toward a redefinition of sex and gender. Amer. Psychol. 34, 11 (1979), 1085.

[57] V. Vinayagamoorthy, M. Gillies, A. Steed, E. Tanguy, X. Pan, C. Loscos, and M. Slater. 2006. Building expression into virtual characters. In Proceedings of the 27th Annual Conference of the European Association for Computer Graphics (Eurographics'06) - State of the Art Reports, Vienna, Austria, September 4-8, 2006. Eurographics Association, 21-61. DOI : https://doi.org/10.2312/egst.20061052

[58] Katja Zibrek, Ludovic Hoyet, Kerstin Ruhland, and Rachel Mcdonnell. 2015. Exploring the effect of motion type and emotions on the perception of gender in virtual humans. ACM Trans. Appl. Percept. 12, 3 (2015), 11.

Received June 2020; accepted August 2020 